\title{
Physical Health Status of Emergency Care Providers in South Africa
}

\author{
Solomon Mthombeni ${ }^{1}$, Yoga Coopoo ${ }^{1}$ and Habib Noorbhai ${ }^{1, *}$ \\ ${ }^{1}$ Department of Sport and Movement Studies, Faculty of Health Sciences, University of Johannesburg, Johannesburg, South Africa \\ "Corresponding author: Department of Sport and Movement Studies, Faculty of Health Sciences, University of Johannesburg, Johannesburg, South Africa. Email: \\ habib.noorbhai@gmail.com
}

Received 2019 December 25; Revised 2020 April 05; Accepted 2020 April 16.

\begin{abstract}
Background: Emergency care providers (ECPs) have a physically, mentally, and emotionally demanding profession. Therefore, they are predisposed to cardiovascular and other non-communicable disease risk factors.

Objectives: The objective of the study was to determine the physical health status of ECPs in the North West province of South Africa through a selected anthropometric and other health parameter test battery.

Methods: Ninety-one ECPs (64 males, 27 females) took part in the study voluntarily for health screening tests including body mass index (BMI), lean body mass (LBM), resting heart rate (RHR), blood pressure (BP), skinfold measurement, waist circumference (WC), waist-to-hip ratio (WHR), fasting blood glucose (FBG), and total cholesterol (TC). The collected data were subjected to statistical analysis using the Statistical Package for Social Sciences (SPSS) version 25 (IBM).

Results: The participants demonstrated a mean BMI of $28.2 \pm 5.5 \mathrm{~kg} / \mathrm{m}^{2}$, body fat of $26 \pm 7.6 \%$, and LBM of $58.6 \pm 10 \mathrm{~kg}$. Significant differences were seen in height (170.5 \pm 6.2 vs. $160.7 \pm 5.3 \mathrm{~cm}$ ), BF\% (22.5 $\pm 5.3 \mathrm{vs.} 34.2 \pm 6.2 \%$ ), and LBM (62.3 $\pm 8 \mathrm{vs.} 49.2 \pm 8.2 \mathrm{~kg}$ ) between males and females $(\mathrm{P} \leq 0.05)$. Mean systolic BP was $122 \pm 15 \mathrm{mmHg}$, and diastolic BP was $81 \pm 10 \mathrm{mmHg}$. Mean WC was 90.8 $\pm 11.4 \mathrm{~cm}$. Other health parameters included mean FBG of $5.1 \pm 2.4 \mathrm{mmol} / \mathrm{L}$ and mean TC of $4.9 \pm 0.7 \mathrm{mmol} / \mathrm{L}$. Significant differences were seen in WHR $(0.88 \pm 0.04$ vs. $0.79 \pm 0.06)$ between males and females $(\mathrm{P} \leq 0.05)$.

Conclusions: A significant number of ECPs presented with cardiovascular and other NCD risk factors such as hypertension, obesity, high WC, elevated FBG, and abnormal levels of TC. This can be attributed to the nature of their occupation such as working irregular shifts leading to sleep deprivation, being exposed to psychological trauma, poor nutrition during shifts, and/or lack of exercise. Stress management is an important part of these workers' rehabilitation program. A well-formulated employee wellness program is required to set remedial measures in place.
\end{abstract}

Keywords: ECPs, Workplace Interventions, Exercise, Clinical Parameters, Cardiovascular Disease

\section{Background}

Emergency care providers (ECPs) have a physically, mentally, and emotionally demanding profession. They are a group of healthcare professionals required to rescue casualties during emergencies as rapidly and efficiently as possible (1). Emergency medical care (EMC) is known as the rescue, evaluation, treatment, and care of injured or ill persons in an emergency, as well as transportation to healthcare facilities (2). Emergency care providers are a group of pre-hospital professionals ranging from basic, intermediate, to advanced life support practitioners registered under section 17 of the professional board of emergency care of the Health Professions Council of South Africa (HPCSA) (3).

Obesity is known to be one of the most significant contributors to hypertension. The obese population is determined not only based on the body mass index (BMI) but also according to other anthropometric indices such as waist circumference (WC), waist-to-hip ratio (WHR), and waist-to-height ratio (WHtR). The WC, WHR, and WHtR indices are important indicators of the central (abdominal) fat distribution, which cannot be determined by BMI. Abnormal central fat distribution, besides BMI, is an important risk factor for hypertension (4). Hypertension is known to be the leading cause of cardiovascular disease, affecting approximately one billion people globally (5). The prevalence of obesity and non-communicable diseases (NCDs) such as hypertension, ischemic heart disease, and type two diabetes is increasing among all population groups, and health care providers are no exception in South Africa (6). Van der Merwe and Pepper (7) indicated that at least $57 \%$ of females and $29 \%$ of adult males in South Africa across all ethnic groups were overweight and obese. Non-communicable diseases account for more than $35 \mathrm{mil}$ lion global deaths per year (8). 
Emergency care providers are exposed to psychosocial stressors induced by traumatic events, repeated incidents within a short time frame, professional conflict, and emotional reactions to ill or near-to-death persons. Exposure to such stressors can cause an increase in physical, physiological, and psychological demands during onduty emergency callouts. Continuous exposure to stressors leads to overactivity of the autonomic nervous system, resulting in the development of mental health impairments, obesity, metabolic syndrome, and cardiovascular disease (1). Irandoust and Taheri (9) indicated that there was a $25 \%$ higher prevalence of depression among obese people than among non-obese individuals. HeggDeloye et al. (10) examined the prevalence of cardiovascular risk factors among paramedics and found that more than $88 \%$ had at least one cardiovascular risk factor. Male paramedics showed more cardiovascular risk factors than female paramedics. The study went further to indicate that $18 \%$ of on-duty fatalities among paramedics were due to cardiovascular events, as reported by other related studies (10). Therefore, despite the increase of NCDs among the South African population, ECPs may be at a higher risk due to the demanding nature of their occupation. Following an evaluation on the occupational risk factors for hypertension and metabolic syndrome, as well as the prevalence of high blood pressure among firefighters, police officers, and paramedics, Kales et al. (11) found that occupational factors that were likely to increase the risk of cardiovascular disease among ECPs included the lack of exercise, poor nutrition (sometimes attributable to limited opportunities for healthy food choices while on duty), shift work (sleep disruption/deprivation), noise exposure, posttraumatic stress disorder, and imbalances between job demands and decisional latitude. When evaluating the physical health of ECPs, their status of physical fitness also needs to be taken into consideration. A study by Bužga et al. (12) found that physical fitness levels among a group of paramedics in the Czech Republic were comparable to those of the general population. This is despite the high physical fitness demand of the paramedic profession to prevent work-related musculoskeletal disorders (13). It was advocated for the establishment of physical fitness criteria for the profession of paramedics to preserve work effectiveness and a good state of health among them. The lack of physical fitness among paramedics may also contribute to poor health and the risk of cardiovascular disease (14). An insufficient number of studies have investigated the physical health status of ECPs in South Africa despite the increased prospects of NCDs associated with their occupation.

\section{Objectives}

The purpose of the study was to determine the health characteristics of ECPs in the North West province of South Africa by measuring some health parameters.

\section{Methods}

\subsection{Participants}

Ninety-one (64 men and 27 women) participants with an age range of 26 to 50 years took part in the study. The total number of participants invited to partake in the study was 127 ECPs.

\subsection{Inclusion Criteria}

Participants were required to have at least two years of work experience as an operational ECP.

\subsection{Study Design}

This was a descriptive cross-sectional study that utilized analytical methods. All participants completed a Physical Activity Readiness Questionnaire (PARQ) before data collection.

\subsection{Data Collection}

Eight parameters were selected to measure the health status of ECPs, including Body Mass Index (BMI), Resting Heart Rate (RHR), Blood Pressure (BP), skinfold measurement, Waist Circumference (WC), Waist-to-Hip Ratio (WHR), Fasting Blood Glucose (FBG), and Total Cholesterol (TC).

\subsubsection{Height and Body Mass}

The height and body mass of the participants were measured to determine the BMI. A Micro A12 Physician scale (Premier scale services) with a vertical ruler was used for the measurements (15).

\subsubsection{Lean Body Mass}

Lean body mass was calculated by measuring the participants' body weight, multiplied by the fat percentage, subtracted from the bodyweight (15).

\subsubsection{Body Mass Index (BMI)}

The purpose of BMI assessment is to categorize a person into either underweight, normal weight, overweight, or obese groups. The BMI of participants was calculated using equation BMI $\left(\mathrm{kg} \cdot \mathrm{m}^{-2}\right)=$ body mass $(\mathrm{kg}) /$ height squared $\left(\mathrm{m}^{2}\right)(15)$. The norms used for BMI in this study are as follows: Less than 18.5 (underweight); 18.5 to 24.9 (normal weight); 25 to 29.9 (overweight); 30 to 34.9 (obesity class one); 35 to 39.9 (obesity class two); and $40>$ (obesity class three) (15). 


\subsubsection{Resting Heart Rate (RHR)}

A Polar FT7 heart rate monitor was used to measure the RHR of the subjects. The heart rate was recorded as beats per minute (bpm) (15).

\subsubsection{Blood Pressure (BP)}

Blood pressure was a demographic measurement of participants. A HI-CARE international blood pressure monitor kit was used to measure the blood pressure of participants (15). The norms used for BP in this study are as follows: Normal (systolic: < 120 and diastolic: $<80 \mathrm{mmHg}$ ); pre-hypertension (systolic: 120 - $139 \mathrm{mmHg}$; diastolic: 80 $89 \mathrm{mmHg}$ ); Hypertension stage 1 (systolic: 140 - $159 \mathrm{mmHg}$; diastolic: 90 - $99 \mathrm{mmHg}$ ); and Hypertension stage 2 (systolic: $160 \mathrm{mmHg}$; diastolic: 80 - $89 \mathrm{mmHg}$ ) (15).

\subsubsection{Skinfolds}

The purpose of the test was to evaluate the subcutaneous fat percentage of participants. A skinfold caliper (Harpenden Baty International) was used for the measurement of skinfolds. The Durnin and Womersley equation was selected to calculate the sum of all skinfolds to determine the total body fat percentage (15). Skinfolds were measured at biceps, triceps, subscapularis, and suprailiac. The norms used for body fat percentage are presented in Table 1.

\subsubsection{Waist Circumference (WC)}

The purpose of the test was to determine the waist size of the participants. High waist circumference is associated with high visceral fat known as abdominal obesity. The waist circumference was measured using a $150 \mathrm{~cm}$ Dynatronics body circumference measuring tape. The tape was placed around the smallest area of the waist, around 2.54 $\mathrm{cm}$ above the navel (15). The norms used for waist circumference in this study are as follows: Normal (males $>102$ $\mathrm{cm}$; females $>88 \mathrm{~cm}$ ); Central obesity (males $<102 \mathrm{~cm}$; females $<88 \mathrm{~cm})(16)$.

\subsubsection{Waist-to-Hip Ratio (WHR)}

The purpose of the test was to determine the circumference of the waist relative to the circumference of the hip as an indicator of body fat distribution. A $150 \mathrm{~cm}$ measuring tape (Dynatronics) was used to measure both the hip and waist circumferences. The WHR was determined by dividing the value of the waist circumference by the value of the hip circumference using a calculator (15). The norms used for body fat percentage are presented in Table 2 .
3.4.9. Fasting Blood Glucose (FBG) and Fasting Total Cholesterol (TC)

A COBAS Accutrend Plus cholesterol, glucose, lactate, and triglyceride machine was used to measure blood glucose and cholesterol of the participants (15). The norms used for FBG in this study are as follows: $<6.1 \mathrm{mmol} / \mathrm{L}$ (nor$\mathrm{mal}$ ); 6.1 - $6.9 \mathrm{mmol} / \mathrm{L}$; and $7.0>\mathrm{mmol} / \mathrm{L}(17)$. The norms used for fasting TC in this study are as follows: 2.7-5.5 (low); 5.6 - 6.7 (moderate); and 6.8 - 14.8 (high) (18).

\subsection{Data Analysis}

All measurements of health parameters were captured on a Microsoft Excel spreadsheet. Both descriptive (means and standard deviation(SD)) and inferential statistics were computed on the Excel spreadsheet from the obtained data. The analysis was used to identify the demographical information and health parameters of ECPs and compare them to available norms for the general adult population. A Student's t-test was used to compare the results between males and females. A statistical software package SPSS version 25 (IBM) was used for the statistical analysis. The level of significance was set at $\mathrm{P}<0.05$.

\subsection{Ethical Considerations}

Ethical approval for the study was obtained from the Research Ethics Committee of the Faculty of Health Sciences, University of Johannesburg (REC-01-159-2016). All participants completed and signed informed consent forms before taking part in the study. The research also complied with the World Medical Association Declaration of Helsinki on Ethical Principles for Research Involving Human Participants (19).

\section{Results}

The demographic data showed a mean age of $36.7 \pm 5.4$ ranging from 26 to 50 years among participants. The majority $(62 \%)$ of the participants were in the age group of 30 -39. The mean height was $167.6 \pm 7.4 \mathrm{~cm}$ (males, $170.5 \pm 6.2$ vs. females, $160.7 \pm 5.3 \mathrm{~cm}$ ). The mean weight was $77 \pm 16.5$ $\mathrm{kg}$ in male participants and $81 \pm 13.8 \mathrm{~kg}$ in female participants.

The health parameters of participants are portrayed in Table 3. Significant differences were seen in height, BF, and LBM between males and females $(P \leq 0.05)$. Significant differences were also seen in the WHR between males and females $(\mathrm{P} \leq 0.05)$. 


\begin{tabular}{|c|c|c|c|c|c|c|c|c|}
\hline \multirow[b]{2}{*}{ Gender } & \multirow[b]{2}{*}{ Male } & \multirow[b]{2}{*}{ Female } & \multirow[b]{2}{*}{ Male } & \multicolumn{2}{|c|}{ Rating } & \multirow[b]{2}{*}{ Female } & \multirow[b]{2}{*}{ Male } & \multirow[b]{2}{*}{ Female } \\
\hline & & & & Female & Male & & & \\
\hline Age Group (Years) & $18-25$ & $18-25$ & $26-35$ & $26-35$ & $36-45$ & $36-45$ & $46-55$ & $46-55$ \\
\hline Very lean (\%) & $4-7$ & $13-17$ & $8-12$ & $13-18$ & $10-14$ & $15-19$ & $12-16$ & $18-22$ \\
\hline Lean (\%) & $8-10$ & $18-20$ & $3-15$ & $19-21$ & $16-18$ & $20-23$ & $18-20$ & $23-25$ \\
\hline Leaner than average (\%) & $11-13$ & $21-23$ & $16-18$ & $22-23$ & $19-21$ & $24-26$ & $21-23$ & $26-28$ \\
\hline Average (\%) & $14-16$ & $24-25$ & $19-21$ & $24-26$ & $22-24$ & $27-29$ & $24-25$ & $29-31$ \\
\hline Slightly high (\%) & $18-20$ & $26-28$ & $22-24$ & $27-30$ & $25-26$ & $30-32$ & $26-28$ & $32-34$ \\
\hline High (\%) & $22-26$ & $29-31$ & $25-28$ & $31-35$ & $27-29$ & $33-36$ & $29-31$ & $36-38$ \\
\hline Very high (\%) & $>28$ & $>33$ & $>30$ & $>36$ & $>30$ & $>39$ & $>32$ & $>39$ \\
\hline
\end{tabular}

${ }^{\text {a }}$ Reprinted [adapted] from Miller (15)

\begin{tabular}{|c|c|c|c|c|c|c|c|c|}
\hline Gender & Males & Females & Males & Females & Males & Females & Males & Females \\
\hline Age group & $20-29$ & $20-29$ & $30-39$ & $30-39$ & $40-49$ & $40-49$ & $50-59$ & $50-59$ \\
\hline Low & $<0.83$ & $<0.71$ & $<0.84$ & $<0.72$ & $<0.88$ & $<0.73$ & $<0.90$ & $<0.74$ \\
\hline Moderate & $0.83-0.88$ & $0.71-0.77$ & $0.84-0.91$ & $0.72-0.78$ & $0.88-0.95$ & $0.73-0.79$ & $0.90-0.96$ & $0.74-0.81$ \\
\hline High & $0.89-0.94$ & $0.78-0.82$ & $0.92-0.96$ & $0.79-0.84$ & $0.96-1.00$ & $0.80-0.87$ & $0.97-1.02$ & $0.82-0.88$ \\
\hline Very high & $>0.94$ & $>0.82$ & $>0.96$ & $>0.84$ & $>1.00$ & $>0.87$ & $>1.02$ & $>0.88$ \\
\hline
\end{tabular}

${ }^{\text {a }}$ Reprinted [adapted] from Miller (15)

Table 3. Health Parameters for Male and Female Emergency Care Providers

\begin{tabular}{|c|c|c|c|c|}
\hline Variables & Combined, Mean \pm SD & Males, Mean \pm SD & Females, Mean \pm SD & Significance \\
\hline BMI $\left(\mathrm{kg} \mathrm{m}^{-2}\right)$ & $28.4 \pm 5$ & $27.9 \pm 4.7$ & $29.6 \pm 5.4$ & 0.201 \\
\hline Body fat $\%$ & $26 \pm 7.6$ & $22.5 \pm 5.3$ & $34.2 \pm 6.2$ & $0.001^{\mathrm{a}}$ \\
\hline Lean body mass (kg) & $58.6 \pm 10$ & $62.3 \pm 8$ & $49.2 \pm 8.2$ & $0.001^{\mathrm{a}}$ \\
\hline Resting heart rate (bpm) & $96.7 \pm 16$ & $97.4 \pm 15.8$ & $94.8 \pm 16.9$ & 0.553 \\
\hline Systolic blood pressure (mmHg) & $122 \pm 15$ & $124 \pm 14$ & $118 \pm 16$ & 0.360 \\
\hline Diastolic blood pressure (mmHg) & $81 \pm 10$ & $82 \pm 10$ & $79 \pm 10$ & 0.305 \\
\hline Waist circumference $(\mathrm{cm})$ & $90.8 \pm 11.4$ & $91.8 \pm 10.7$ & $88.2 \pm 12.3$ & 0.130 \\
\hline Waist-to-hip ratio & $0.85 \pm 0.06$ & $0.88 \pm 0.04$ & $0.79 \pm 0.06$ & $0.001^{\mathrm{a}}$ \\
\hline Fasting blood glucose $(\mathrm{mmol} / \mathrm{L})$ & $5.1 \pm 2.4$ & $5.4 \pm 2.7$ & $4.6 \pm 1$ & 0.186 \\
\hline Fasting total cholesterol $(\mathrm{mmol} / \mathrm{L})$ & $4.9 \pm 0.7$ & $4.88 \pm 1$ & $4.7 \pm 0.73$ & 0.567 \\
\hline
\end{tabular}

Abbreviations: BMI, body mass index; cm, centimeters; kg, kilogram; mmHg, millimeters of mercury; mmol/L, millimole per liter; SD, standard deviation, ${ }^{\mathrm{a}} \mathrm{P} \leq 0.05$

\section{Discussion}

The purpose of the study was to determine the physical health characteristics of ECPs in the North West province of South Africa based on some selected health parameters. The participants were required to complete the PARQ for gathering information about their health history and physical activity status. A total of $8 \%$ of ECPs reported that they were current smokers or ceased smoking within six months before answering the questionnaire. The prevalence of diagnosed hypertension reported by ECPs was $8 \%$, with only $3.4 \%$ reporting being diagnosed with hypercholesterolemia. However, the study found that $24 \%$ of the participants had hypertension (16\% stage I and $8 \%$ stage II), and 2.3\% had hypercholesterolemia (total cholesterol > 
$7.5 \mathrm{mmol} / \mathrm{L}$ ). In the PARQ, 59.7\% of the ECPs indicated that they engaged in occasional physical activity (recreational sports activities) averaging twice per week. However, the survey did not include a question regarding the duration and intensity of physical activities. Swain et al. (16) indicated that smoking, hypertension, and lack of regular physical activity are risk factors for myocardial infarction in both men and women of all ages. The impact of hypertension on ECPs can be seen in their work, life, and activities. These implications are far-reaching, with a negative link to their body weight, which could result in obesity over time. According to Dalal et al. (20), RHR is one of the factors associated with incident hypertension. Elevated RHR is a risk factor, not just a risk indicator. The heart rate alongside hypertension tends to be elevated, and it has been identified as an independent predictor for adverse cardiovascular and mortality outcomes, which can aid in determining the prognosis of persons with high BP (20). This should also be taken into consideration when managing programs with ECPs.

There was no significant difference in age, body weight, and BMI between genders. In terms of BMI, a total of $2.29 \%$ of the participants were classified as underweight (BMI $<18.5$ ), 26.43\% as normal weight (BMI 18.5 - 24.9), 37.93\% as overweight (BMI 25 - 29.9), and 33.33\% as obese (BMI $>30$ ). These findings are consistent with Tsismenakis et al. (21) study that found $0.8 \%$ underweight, $22.4 \%$ normal weight, $43.8 \%$ overweight, and 33\% obese in a group of 160 paramedics/emergency care technicians and 270 firefighter candidates. Hegg-Deloye et al. (1) found a similar average BMI (28.2 \pm 5.6$)$ in a group of 295 paramedics. Overweight and obesity in the work environment are associated with occupational risks such as musculoskeletal disorders and injury, asthma, immune responses, cardiovascular disease, neurotoxicity, stress, and cancer (22). Irandoust et al. (23) also indicated that weight gain among adults in their middle ages could have certain metabolic effects leading to consequences such as depression, sleep disruption, and other psychological disorders. Weight gain by ECPs overtime not only may present work-related musculoskeletal disorders, but also could negatively affect their mental health and wellbeing.

The average time that ECPs reported engaging in physical activity was below the American College of Sports Medicine (ACSM) recommendations of at least 30 - $60 \mathrm{~min}$ of moderate-intensity exercise five days per week for adults and 30 minutes of physical activity for most if not all days of the week (16). Reducing overweight and obesity in ECPs requires multiple approaches such as promoting physical fitness, making BMI as one of the vital signs during medical examinations of candidates, ensuring commitment from labor and management to maintain health and fitness pro- grams, the establishment of workplace fitness facilities, and compulsory exercise sessions during working hours (21). Exercise sessions during working hours can be classified as a means of occupational fitness (24). According to Tipton et al. (24), occupational fitness standards for emergency services have been based on tasks that ensure the safety and well-being of the public, fellow workers, and individuals. As such, it is imperative to bear in mind that high inactivity levels can result in poor occupational fitness. Specifically, certain critical tasks that ECPs fail to perform to an acceptable standard could endanger the workforce, individual, or team (25).

Aside from physical inactivity, it is also important to address the BF percentage findings in the study. In this study, the BF percentage was classified according to norms by Swain et al. (16), which indicated that $29.8 \%$ of ECPs had low $\mathrm{BF}, 16 \%$ average $\mathrm{BF}, 44.8 \%$ high $\mathrm{BF}$, and $9.2 \%$ very high $\mathrm{BF}$. Patel and Abate (26) suggested that people with high subcutaneous and visceral body fat content (even those with normal BMI) had a high prevalence of cardio-metabolic dysregulation, cardiovascular risk factors, and metabolic syndrome. Body fat is stored in adipose tissue. The study indicated that metabolic abnormalities and insulin resistance can be due to differential distribution and dysfunction of adipose tissue. Adipose tissues are divided into the central (abdominal) region and peripheral (upper and lower extremities) region. High BF may negatively impact not only the health of ECPs but also their work-related capabilities. Chapman et al. (27) demonstrated that the BF percentage of ECPs could be just as important as aerobic capacity. Most job-related tasks of ECPs such as patient handling and cardiopulmonary resuscitation (CPR) require maximum aerobic levels. A reduced body fat percentage could lead to reduced intensity and effort for such activities. The increased intensity and effort may lead to premature fatigue and ineffective execution of rescue emergency care duties. Therefore, the regular screening of body composition is necessary for ECPs to ensure a high quality of care. Furthermore, as stated above for physical inactivity, an acceptable level and range of body fat percentage must be addressed for ECPs to maintain an adequate level of occupational fitness in the emergency services fraternity.

In addition to the BF percentage, the RHR of the participants ( $96.7 \pm 16 \mathrm{bpm}$ ) was higher than the normal values of between 60 and $80 \mathrm{bpm}$. The SBP and DBP measurements were classified according to the norms by Swain et al. (16). It was found that $39 \%$ of the participants had normal BP (< $120 \mathrm{mmHg}$ systolic, < $80 \mathrm{mmHg}$ diastolic), 36\% pre-hypertension (120 - 139 mmHg systolic, 80 - 89 mmHg diastolic), 16\% stage I hypertension (140 - $159 \mathrm{mmHg}$ systolic, 90 - $99 \mathrm{mmHg}$ diastolic), and 8\% stage II hypertension (> $160 \mathrm{mmHg}$ systolic, > $100 \mathrm{mmHg}$ diastolic). Kales 
et al. (11) found that the prevalence of high blood pressure among emergency responders grew with age and that obesity was yet another important factor increasing the prevalence of hypertension among them. Houston (5) indicated a strong association between hypertension and increased risk of morbidity and mortality from cerebrovascular accidents (stroke), congestive heart failure, coronary heart disease, and end-stage renal failure. Hypertension may be reversed by regular exercise, adequate nutrition, and stress coping mechanisms (10). A study by Houston (5) shows that diet plays an important role in stabilizing BP. Dietary interventions known to manage and/or lower BP include: reduced sodium intake, increased magnesium, potassium, and calcium intake, and using diets rich in fruits and vegetables. The management of hypertension among ECPs may require healthy diet intervention and obesity reduction.

A risk status must be assessed by determining the degree of overweight or obesity based on BMI, the presence of abdominal obesity based on WC, and the presence of concomitant cardiovascular risk factors or comorbidities (28). Aside from a high level of obesity rates, BMI, BF percentage, $\mathrm{BP}$, or RHR, a high WHR ratio has also been recognized as a risk factor for disease and is known to be a better predictor of mortality than BMI (16). Accordingly, 24.13\% of the participants showed a low WHR, 49.4\% moderate WHR, 19.5\% high WHR, and $6.9 \%$ very high WHR. The study also found that $14.3 \%$ of males and $41.7 \%$ of females had increased WC (abdominal obesity/high visceral fat). Increased WC is known to be a major risk factor for hypokinetic diseases such as cardiovascular disease, dyslipidemia, and insulin resistance $(16,29)$. Similar health parameters are discussed together to determine the level of risk in this population. Based on Patel and Abate (26) findings of epidemiological studies assessing regional adiposity, an increase in WHR is associated with impaired glucose tolerance, hyperinsulinemia, hypertriglyceridemia, hypercholesterolemia, hyperuricemia, type two diabetes, and atherosclerosis. A study by Irandoust et al. (23) also found that increased abdominal fat accumulation was associated with symptoms of depression among adults. Their study suggested that exercise intervention could decrease overall $\mathrm{BF}$, therefore leading to depression reduction. That exercise has beneficial effects on depression is based on the fact that physical activity increases the serotonin and norepinephrine levels during exercise training and makes adaptations resulting from reduced cortisol levels and enhanced androgen release, therefore lowering depression. A reduction in $\mathrm{BF}$ of ECPs, both peripheral and central, may assist in NCD risk reduction and improved psychological wellbeing.

Fasting blood glucose and TC formed part of health parameters in the study. The study found that $64.4 \%$ of the participants presented with low to normal FBG $(<5.0)$, $31 \%$ had impaired fasting glucose (IFG), $2.3 \%$ presented with Impaired Glucose Tolerance (IGT), and $2.3 \%$ had values classified as diabetes. Uncontrolled IFG and IGT may lead to diabetes over time. Fasting TC values for participants showed that $64.4 \%$ had low levels of fasting TC and $35.6 \%$ had moderate to high levels. High cholesterol levels are known to have a strong association with coronary artery disease, which can lead to cardiovascular disease and stroke (16). Therefore, ECPs are required to obtain normal levels of total cholesterol to reduce their risk factors for cardiovascular disease through regular physical activity and workplace-specific nutrition education and training programs (30). More impact on health and NCDs is needed, especially for occupational health.

The majority of the health parameters measured in the study were associated with risk factors for NCDs and the majority of ECPs presented with one or more risk factors. Elevated BP, obesity, advanced age, insulin resistance, dyslipidemia, and glucose intolerance in ECPs are the risk factors of cardiovascular disease (11). Despite that the study did not evaluate the nutritional intake of ECPs, it is important to understand that nutrition plays an important role in the overall health of individuals. Wu et al. (31) indicated that behavioral factors such as unhealthy diet, tobacco use, lack of physical activity, and excessive alcohol use are known and modifiable contributors to several health mediators of NCDs. Kales et al. (11) also indicated that one of the occupational risk factors of cardiovascular disease among ECPs was poor nutrition while on duty. The increase in the NCD risk among ECPs is further exacerbated by the strenuous nature of their work leading to various psychosocial stressors, as indicated by HeggDeloye et al. (10). Long-term consequences of NCDs and other work-related musculoskeletal injuries among ECPs may present with poor health outcomes leading to workplace absenteeism, early retirement, inability to execute occupational duties, increased medical expenses, or even premature death.

\subsection{Conclusions}

A significant number of ECPs presented with cardiovascular and other NCD risk factors such as hypertension, obesity, high waist circumference, elevated fasting blood glucose, and abnormal levels of total cholesterol. This can be attributed to the nature of their occupation such as working irregular shifts leading to sleep deprivation, being exposed to psychological trauma leading to posttraumatic stress disorders, poor nutrition during shifts, and/or lack of exercise. The cities in which these health care providers are working need to provide employee assistance programs for them to set remedial plans in place for 
healthy eating, education, fitness, and stress-related counseling. Fitness and health assessments must be conducted at least every year with remedial programs put in place at an onsite health and wellness center. These programs will create health and wellness awareness and education for a better, healthier, and fitter emergency care provider.

\subsection{Recommendations}

Annual health screening programs in the form of wellness day services for ECPs should be implemented given the prevalence of cardiovascular and metabolic syndrome risk factors. Such programs will lead to improvements in overall health awareness among ECPs and encourage them to consider preventative measures.

\subsection{Limitations}

The limitation of this study was that the participants sampled were all from one province in South Africa, and it shows trends in this occupation, and the results should not be generalized to the whole country. Further studies are required to acquire a national occupational picture. The study also presents another limitation as it did not evaluate the psychological and nutritional aspects of ECPs despite their strong impact on overall health. Future studies should include the evaluation of psychological (such as by mood states test) and nutritional impacts on the health status of ECPs. Furthermore, future studies should include broader lipid profile measurements aside from blood glucose and cholesterol.

\section{Footnotes}

Authors' Contribution: SM conducted data collection and wrote the paper. YC conceptualised the study, assisted with data analysis and the write-up of the paper. $\mathrm{HN}$ assisted with the formulation and write-up of the paper.

\section{Conflict of Interests: None.}

Ethical Approval: Ethical approval for the study was obtained from the research ethics committee at the Faculty of Health Sciences, University of Johannesburg (REC-01-1592016). The research also complied with the World Medical Association Declaration of Helsinki on Ethical Principles for research involving Human Participants.

\section{Funding/Support: None.}

Informed Consent: All participants completed and signed an informed consent prior to taking part in the study.

\section{References}

1. Hegg Deloye S. Work-related and Dietary Factors Associated with Weight Gain over the Period of Employment in Paramedics. Occupational Medicine \& Health Affairs. 2014;2(4). doi: 10.4172/23296879.1000173.

Asian J Sports Med. 2020; 11(2):e100261.
2. Gulli B, Ciatolla JA, Barnes L. Emergency care and transportation of the sick and injured. 8th ed. Jones \& Bartlett Learning; 2011.

3. Wallis LA, Garach SR, Kropman A. State of emergency medicine in South Africa. Int J Emerg Med. 2008;1(2):69-71. doi: 10.1007/s12245-0080033-3. [PubMed: 19384654]. [PubMed Central: PMC2657239].

4. Lee JW, Lim NK, Baek TH, Park SH, Park HY. Anthropometric indices as predictors of hypertension among men and women aged 40-69 years in the Korean population: the Korean Genome and Epidemiology Study. BMC Public Health. 2015;15:140. doi: 10.1186/s12889-015-1471-5. [PubMed: 25886025]. [PubMed Central: PMC4332746].

5. Houston $M$. The role of magnesium in hypertension and cardiovascular disease. J Clin Hypertens (Greenwich). 2011;13(11):843-7. doi: 10.1111/j.1751-7176.2011.00538.x. [PubMed: 22051430].

6. Kruger HS, Puoane T, Senekal M, van der Merwe MT. Obesity in South Africa: challenges for government and health professionals. Public Health Nutr. 2005;8(5):491-500. doi: 10.1079/phn2005785. [PubMed: 16153330].

7. van der Merwe MT, Pepper MS. Obesity in South Africa. Obes Rev. 2006;7(4):315-22. doi: 10.1111/j.1467-789X.2006.00237.x. [PubMed: 17038125].

8. Kontis V, Mathers CD, Rehm J, Stevens GA, Shield KD, Bonita R, et al. Contribution of six risk factors to achieving the $25 \times 25$ non-communicable disease mortality reduction target: a modelling study. Lancet. 2014;384(9941):427-37. doi: 10.1016/S01406736(14)60616-4. [PubMed: 24797573].

9. Irandoust K, Taheri M. The Effect of Vitamin D supplement and Indoor Vs Outdoor Physical Activity on Depression of Obese Depressed Women. Asian Journal of Sports Medicine. 2017;In Press(In Press). doi: 10.5812/asjsm.13311.

10. Hegg-Deloye S, Brassard P, Prairie J, Larouche D, Jauvin N, Poirier P, et al. Prevalence of risk factors for cardiovascular disease in paramedics. Int Arch Occup Environ Health. 2015;88(7):973-80. doi: 10.1007/s00420015-1028-z. [PubMed: 25655920].

11. Kales SN, Tsismenakis AJ, Zhang C, Soteriades ES. Blood pressure in firefighters, police officers, and other emergency responders. Am J Hypertens. 2009;22(1):11-20. doi: 10.1038/ajh.2008.296. [PubMed: 18927545].

12. Bužga M, Jirák Z, Bužgová R. State of physical health and fitness of paramedics in Czech republic. WULFENIA. 2015;22(3).

13. Hunter JR, Macquarrie AJ, Sheridan SC. Physical capacity of New South Wales ambulance paramedics. Occup Med (Lond). 2019;69(8-9):53440. doi: 10.1093/occmed/kqz131. [PubMed: 31605617].

14. Sheridan S. Paramedic health status, fitness and physical tasks: A review of the literature. Australasian Journal of Paramedicine. 2019;16. doi: 10.33151/ajp.16.580.

15. Miller TA. NSCA's Guide to Tests and Assessments. Washington: Human Kinetics; 2012.

16. Swain DP, Brawner CA; American College of Sports Medicine. ACSM's resource manual for guidelines for exercise testing and prescription. Wolters Kluwer Health/Lippincott Williams \& Wilkins; 2014

17. Diabetes.co.uk. The Global Diabetes Community, 2017, Blood Glucose Norms. 2017, [cited 14 November 2017]. Available from: https://www. Diabetes.co.uk/riskfactorsfordiabetes/.

18. Langsted A, Freiberg JJ, Nordestgaard BG. Fasting and nonfasting lipid levels: influence of normal food intake on lipids, lipoproteins, apolipoproteins, and cardiovascular risk prediction. Circulation. 2008;118(20):2047-56. doi: 10.1161/CIRCULATIONAHA.108.804146. [PubMed: 18955664].

19. General Assembly of the World Medical Association. Declaration of Helsinki. Ethical Principles for Medical Research Involving Human Subjects. Jahrbuch für Wissenschaft und Ethik. 2009;14(1). doi: $10.1515 / 9783110208856.233$ 
20. Dalal J, Dasbiswas A, Sathyamurthy I, Maddury SR, Kerkar P, Bansal S, et al. Heart Rate in Hypertension: Review and Expert Opinion. Int J Hypertens. 2019;2019:2087064. doi: 10.1155/2019/2087064. [PubMed: 30915238]. [PubMed Central: PMC6399539].

21. Tsismenakis AJ, Christophi CA, Burress JW, Kinney AM, Kim M, Kales $\mathrm{SN}$. The obesity epidemic and future emergency responders. Obesity (Silver Spring). 2009;17(8):1648-50. doi:10.1038/oby.2009.63. [PubMed: 19300435].

22. Anderson LM, Quinn TA, Glanz K, Ramirez G, Kahwati LC, Johnson $\mathrm{DB}$, et al. The effectiveness of worksite nutrition and physical activity interventions for controlling employee overweight and obesity: a systematic review. Am J Prev Med. 2009;37(4):340-57. doi: 10.1016/j.amepre.2009.07.003. [PubMed: 19765507].

23. Irandoust $\mathrm{K}$, Taheri M, Chtourou H, Nikolaidis PT, Rosemann T, Knechtle B. Effect of Time-of-Day-Exercise in Group Settings on Level of Mood and Depression of Former Elite Male Athletes. Int J Environ Res Public Health. 2019;16(19). doi: 10.3390/ijerph16193541. [PubMed: 31546685]. [PubMed Central: PMC6801561].

24. Tipton MJ, Milligan GS, Reilly TJ. Physiological employment standards I. Occupational fitness standards: objectively subjective? Eur J Appl Physiol. 2013;113(10):2435-46. doi: 10.1007/s00421-012-2569-4. [PubMed: 23263741].

25. Bilzon JL, Scarpello EG, Smith CV, Ravenhill NA, Rayson MP. Characterization of the metabolic demands of simulated shipboard Royal Navy fire-fighting tasks. Ergonomics. 2001;44(8):766-80. doi: 10.1080/00140130118253. [PubMed: 11450875].

26. Patel $\mathrm{P}$, Abate $\mathrm{N}$. Role of subcutaneous adipose tissue in the pathogenesis of insulin resistance. J Obes. 2013;2013:489187. doi: 10.1155/2013/489187. [PubMed: 23691287]. [PubMed Central: PMC3649613].

27. Chapman D, Peiffer J, Abbiss C, Laursen P. A Descriptive Physical Profile of Western Australian Male Paramedics. Australasian Journal of Paramedicine. 2015;5(1). doi: 10.33151/ajp.5.1.403.

28. Expert Panel on the Identification Treatment of Overweight Obesity in Adults; National Heart Lung Blood Institute; National Institute of Diabetes Digestive Kidney Diseases. Clinical guidelines on the identification, evaluation, and treatment of overweight and obesity in adults: the evidence report. National Institutes of Health, National Heart, Lung, and Blood Institute; 1998.

29. Kuk JL, Katzmarzyk PT, Nichaman MZ, Church TS, Blair SN, Ross R. Visceral fat is an independent predictor of all-cause mortality in men Obesity (Silver Spring). 2006;14(2):336-41. doi: 10.1038/oby.2006.43. [PubMed: 16571861].

30. Noorbhai MH. A public health approach to increase physical activity and health education: The Biokinetic Humanitarian Project. African Journal for Physical Health Education, Recreation and Dance. 2013;19(Issue-4_2):993-8.

31. Wu F, Guo Y, Chatterji S, Zheng Y, Naidoo N, Jiang Y, et al. Common risk factors for chronic non-communicable diseases among older adults in China, Ghana, Mexico, India, Russia and South Africa: the study on global AGEing and adult health (SAGE) wave 1. BMC Public Health. 2015;15:88. doi: 10.1186/s12889-015-1407-0. [PubMed: 25885218] [PubMed Central: PMC4335695]. 